\title{
Cardiac Valvular Stenosis Severity
}

National Cancer Institute

\section{Source}

National Cancer Institute. Cardiac Valvular Stenosis Severity. NCI Thesaurus. Code C102245.

The qualitative measurement of the severity of cardiac valvular stenosis. 